\title{
Retraction Note to: Expression and prognostic value of the aldehyde dehydrogenase 1 (ALDH1) and N-myc downstream regulated gene 2 (NDRG2) as potential markers in human astrocytomas
}

\author{
Peyman Karimi Goudarzi ${ }^{1}$ - Farzad Mehrabi ${ }^{2}$ • Reza Jalili Khoshnood ${ }^{3}$. \\ Ali Baradaran Bagheri ${ }^{4} \cdot$ Koorosh Ahmadi $^{5}$ Emad Yahaghi ${ }^{6}$. \\ Hesam Abdolhoseinpour ${ }^{7}$
}

Published online: 5 November 2016

(C) International Society of Oncology and BioMarkers (ISOBM) 2016

Retraction Note to: Tumor Biol (2016) 37:6261-6265

DOI 10.1007/s13277-015-4491-y

This article has been retracted at the request of the Editorin-Chief, the International Society of Oncology and BioMarkers (ISOBM) and the Publisher per the Committee on Publication Ethics guidelines. The article shows evidence of irregularities in authorship and in the materials and methods during the submission process and there is strong reason to believe that the peer review process was compromised.

As such the validity of the content of this article cannot be verified.

The online version of the original article can be found at http://dx.doi. org/10.1007/s13277-015-4491-y.

Hesam Abdolhoseinpour

abdolhosseinpoor@yahoo.com

1 Department of Neurosurgery, AJA University of Medical Sciences, Tehran, Iran

2 Department of Neurology, AJA University of Medical Sciences, Tehran, Iran

3 Department of Neurosurgery, Shahid Beheshti University of Medical Sciences, Tehran, Iran

4 Department of Neurosurgery, Alborz University of Medical Sciences, Karaj, Iran

5 Department of Emergency Medicine, Alborz University of Medical Sciences, Karaj, Iran

6 Department of Molecular Biology, Baqiyatallah University of Medical Sciences, Tehran, Iran

7 Department of Neurosurgery, Bou Ali Hospital, Tehran Medical Sciences Branch, Islamic Azad University, Tehran, Iran 